\title{
Assessing paediatric scald injuries using Laser Speckle Contrast Imaging
}

Filip Lindahl, Erik Tesselaar and Folke Sjöberg

\author{
Linköping University Post Print
}

\section{Tweet}

N.B.: When citing this work, cite the original article.

Original Publication:

Filip Lindahl, Erik Tesselaar and Folke Sjöberg, Assessing paediatric scald injuries using Laser Speckle Contrast Imaging, 2012, Burns, (39), 4, 662-666.

http://dx.doi.org/10.1016/j.burns.2012.09.018

Copyright: Elsevier

http://www.elsevier.com/

Postprint available at: Linköping University Electronic Press

http://urn.kb.se/resolve?urn=urn:nbn:se:liu:diva-90361 


\section{Assessing paediatric scald injuries using Laser Speckle Contrast Imaging}

Filip Lindahl $^{1}$, Erik Tesselaar ${ }^{1}$, Folke Sjöberg ${ }^{1,2,3}$

1. Department of Clinical and Experimental Medicine, Faculty of Health Sciences, Linköping University, Linköping, Sweden

2. Department of Plastic Surgery, Hand Surgery, and Burns, County Council of Östergötland, Linköping, Sweden

3. Department of Anaesthesia and Intensive care, County Council of Östergötland, Linköping, Sweden

Corresponding author:

Erik Tesselaar, Ph.D.

Department of Clinical and Experimental Medicine

Faculty of Health Sciences

Linköping University

SE-58185 Linköping, Sweden

Phone: + 4610 - 1033756

E-mail: erik.tesselaar@liu.se 


\begin{abstract}
Background: The use of objective methods for assessment of burns is limited. Laser Speckle Contrast Imaging (LSCI) is a non-invasive technique for instant measurement of tissue perfusion, making it potentially valuable for early prediction of burn wound outcome.

Aim: To evaluate the influence of technical factors on perfusion and to measure perfusion in burns $0-14$ days post-burn and compare this with the outcome of the burn wound at 14 days postburn.
\end{abstract}

Method: The effect of room light, camera distance and camera angle was studied using a suspension of polystyrene particles. LSCI measurements were performed on 45 scald burns and 32 uninjured areas 0-14 days post-burn.

Result: Technical factors had no clinically relevant effect on measured perfusion. Burns that healed within 14 days had a higher perfusion during the first week post-burn than burns that healed after 14 days or required surgery. The difference in perfusion was largest 4-7 days postburn.

Conclusion: LSCI allows for robust, instant measurement of burns and can easily be applied in a clinical setting. Differences in perfusion during the first week post-burn are related to the outcome after 14 days.

Keywords: scald burns, perfusion, laser speckle contrast imaging, burn depth 
Introduction

Clinical evaluation is the principal method for the estimation of depth of burns. It is a subjective assessment based on visual and tactile inspection of the wound, which is immediate, easily performed and cost-effective [1]. However, even experienced burn surgeons make an inaccurate prognosis in about 25\% of the cases [2]. Therefore, there is a need for reliable techniques in the early prediction of burn wound depth. Such techniques would facilitate choosing appropriate treatment and thereby improve wound healing potential, shorten hospital stay and reduce associated costs [3]. Ideally the technique should be noninvasive, easy to use, inexpensive and without risk for the patient.

Superficial partial burns have an increased blood flow compared to uninjured skin whilst the perfusion in deep partial burns is reduced. Several methods that estimate burn wound depth by measuring perfusion in the injured skin area have been studied. The most evaluated of noninvasive, optical burn assessment techniques is Laser Doppler Imaging (LDI) [1, 4]. Burn wound perfusion as measured with LDI has a good correlation with histology, but it requires measurement times of typically several minutes. This makes the technique prone to movement artefacts and less suitable in unsedated children [5].

In recent years, laser speckle contrast techniques have been gaining increasing attention as tools to assess microcirculatory changes non-invasively. These techniques allow for instantaneous measurement of the microvascular perfusion in large areas of skin, making them promising for assessment of burn wounds. In laser speckle contrast imaging (LSCI), coherent laser light illuminates the skin area of interest. The laser light is randomly backscattered from the optically rough red blood cells (RBCs) and creates an interference pattern or speckle pattern on the camera sensor. When RBCs move, the alterations in the speckle pattern during the exposure time of the camera result in spatial blurring of the image. The level of blurring correlates with the level of movement of RBCs [6]. 
We hypothesised that LSCI provides an easy-to-use and reliable technique to measure perfusion in burn wounds, which may facilitate the prediction of burn wound outcome. Therefore, we measured perfusion in paediatric scald burn wounds at 0-14 days post-burn, and studied the relation with the outcome after 14 days of conservative treatment (ref 14 deep partial). Also, we evaluated the effect of camera distance and angle and room light on the perfusion value generated by the LSCI system, as these factors are relevant when the technique is to be applied for the assessment of burn wounds.

Methods

\section{$\underline{\text { Subjects }}$}

During a five-month period all paedriatic patients with scald burns from the burn intensive care unit at Linköping University Hospital were asked to participate in the study. In total 14 patients (8 female) were included. Their mean age was 3.1 years (median 2, range 0-13). All patients were treated according to normal clinical practice. Since all the included patients were underaged their caregivers were informed about the project and signed a consent form for participation in the study. The study obtained approval from the Regional Ethics Board and was conducted in compliance with the "Ethical principles for medical research involving human subjects" of the Helsinki Declaration.

\section{Technology}

A Laser Speckle Contrast imager (Pericam PSI System, Perimed AB, Järfälla, Sweden) was used to measure skin perfusion. The system uses a divergent laser beam with a wavelength of $785 \mathrm{~nm}$ and has two cameras, of which one captures a speckle contrast image while the other captures a conventional colour image of the measurement area. The perfusion is calculated in each pixel of the speckle contrast image. The image size was set to correspond to a 12 x $12 \mathrm{~cm}$ area of skin and the acquisition rate was set to 21 images/s. With each measurement, the perfusion data from 42 
consecutive images were averaged, resulting in a total measurement time of 2 seconds for each image.

Perfusion images are analysed by calculating mean perfusion levels in regions of the image using a computer program (PimSoft 1.3, Perimed AB, Järfalla, Sweden). The spatial resolution of the perfusion image is $0.2 \mathrm{~mm} /$ pixel at a measurement distance of $25 \mathrm{~cm}$.

\section{Protocol}

The first part of the study was a methodological evaluation of LSCI. The effects of environmental light, camera distance (15 - $36 \mathrm{~cm})$ and camera angle (0 - 45 degrees incident angle) on the perfusion values were independently investigated by imaging the surface of a calibration liquid (Motility Standard, Perimed AB, Järfalla, Sweden). Also, the reproducibility of the instrument was measured by taking 10 sequential images of the surface of the calibration fluid. The calibration fluid is a colloidal suspension of polystyrene particles and is used to calibrate laser Doppler and laser Speckle system, as it gives a constant perfusion value. Light conditions were varied as follows: (1) camera covered with a cloth, (2) windows covered by curtains, ceiling lights off, (3) covered windows, lights on and (4) uncovered windows, lights on.

In the second part of the study, LSCI images were taken from patients consecutively admitted to the burn unit with scald burns at different days post-burn (0-14 days), depending on when patients were scheduled for visit to the burn clinic. LSCI images were captured directly after debridement, which was done under anaesthesia. In total, 95 regions of interest were analysed, of which 32 were control images from uninjured skin, 45 were from injured skin areas that healed within 14 days, and 18 were from injured skin areas that healed after 14 days or required surgery. In each region of interest, the mean perfusion value was calculated.

\section{Data analysis}


Data in text and figures are presented as mean values \pm SD. For the data acquired 0-24 hours post-burn, differences in perfusion were analysed using confidence intervals because of the limited number of burns included. Changes in perfusion over time, and differences between uninjured skin, burns that healed within 14 days and burns that healed after 14 days or required surgery were analysed using one-way analyses of variance. Statistical calculations were done using Microsoft Excel 2007 and GraphPad Prism version 5.0d for Mac OS X (GraphPad Software, San Diego California USA, “www.graphpad.com”). For all analyses, probabilities of less than 0.05 were accepted as significant.

\section{Results}

\section{$\underline{\text { Technical factors }}$}

The coefficient of variance (\% CV) of the perfusion value, when measured repeatedly on a container with calibration fluid, was $0.9 \%$ (mean \pm SD, $338 \pm 3.1$ PU). Perfusion values varied significantly with distance of the camera head (Figure 1A, analysis of variance, $\mathrm{p}<0.001$ ), but there was no significant variation in perfusion within the range $18-27 \mathrm{~cm}(\mathrm{p}=0.68)$. The measured perfusion values were not dependent on the incident angle $(\mathrm{p}=0.59$, Figure 1B). Different lighting conditions resulted in a non-significant $1.8 \%$ variation in perfusion values ( $p=0.34$, Figure $1 \mathrm{C}$ ).

\section{$\underline{\text { Patient example }}$}

Figure 2 shows a series of typical perfusion images of a scald burn at different days post-burn, along with intensity images obtained from the same device. At 3 days post-burn (A), the perfusion is highest in the peripheral parts and decreases radially towards the central parts of the burn (Figure 3). Initially, the perfusion in the central parts is increasing while a decrease is seen in the peripheral parts as the burn heals. At 21 days post-burn (F), perfusion is similar in central and peripheral areas. 


\section{Patient study}

The mean ( \pm SD) perfusion of uninjured skin, of burns that healed within 14 days and of wounds that healed after 14 days or required surgery is shown in Figure 4 for different days post-burn (024 hours, 1-3 days, 4-7 days and 8-14 days post-burn).

The mean perfusion in uninjured skin was $83 \pm 17$ PU 0-24 hours post-burn, and did not change during 21 days post-burn ( $\mathrm{p}=0.98)$.

The mean perfusion in burns that healed within 14 days was $205 \pm 46$ PU 0-24 hours post-burn and increased between 1 and 3 days (236 \pm 92 PU) and 4 and 7 days (351 \pm 152 PU) post-burn. This was followed by a decrease in the second week post-burn (177 $\pm 74 \mathrm{PU})$. In the second week post-burn, perfusion was still significantly higher than in healthy skin $(\mathrm{p}=0.006)$.

In burns that healed after 14 days or required surgery, only a slight increase in perfusion was seen in the first week (0-24 hours: $82 \pm 11$ PU, 1-3 days: $95 \pm 12$ PU, 4-7 days: $108 \pm 33$ ) and a stronger increase in perfusion was not noticed until during the second week post-burn (182 \pm 111 PU). In these burns, the perfusion was not significantly different from uninjured skin.

During 0-24 hours post-burn, only 2 burns were measured that eventually required surgery after 14 days. These burns had a mean perfusion of $74 \mathrm{PU}$ and $90 \mathrm{PU}$, respectively. In both cases, their perfusion was below the lower confidence limit of the perfusion in those burns that would heal within 14 days (mean: 295; 95\%CI: 115 to 295 PU). Since we included only 2 burns that healed after 14 days or required surgery, we could not show a significant difference between these burns and burns that healed within 14 days.

Between 1-3 and 4-7 days post-burn, the mean perfusion in burns that healed within 14 days was significantly higher than in burns that healed after 14 days or required surgery $(\mathrm{p}<0.05)$ and compared with uninjured skin $(\mathrm{p}<0.01)$. The difference in perfusion between these two groups 
was largest between 4-7 days post-burn. During the second week post-burn, there was no significant difference in perfusion between any of the groups.

\section{Discussion}

LSCI is a noninvasive, camera-based technique that is recently commercialised. LSCI is comparable to laser Doppler imaging (LDI) in that it measures tissue perfusion by means of laser light. An important advantage of LSCI compared with LDI is the short time needed for a measurement as compared traditional laser Doppler Imaging [4]. With LSCI, it is possible to obtain a complete perfusion image within a few seconds, which makes it particularly suitable for use in patients that are not able to lie still very long, such as young children.

To our best knowledge, this is the first study in which LSCI is used for the assessment of burns. The main finding in this study is that LSCI is a robust and easy to use method that can reliably measure changes in the perfusion of scald burns during the first weeks post-burn. During the first week, perfusion was higher for those wounds that healed within 14 days compared with those that required surgery, which was done at 14 days post-burn. The difference was most pronounced between 4-7 days post-burn but was already significant after one day post-burn. Wounds that heal after 14 days or require surgery have a consistently low perfusion value (below 100 A.U., similar to uninjured skin). Since absolute perfusion values are visualized using fixed colors, the use of an absolute color scale to assess burn wounds seems feasible.

These findings suggest that LSCI can be used as a method to predict burn outcome, at least in the population of patients that was studied, children with superficial or deep partial thickness scald burns.

\section{$\underline{\text { Technical evaluation }}$}


The effect on technical factors on our burn wound measurements indicate that the technique is very robust to variations in distance between camera head and tissue, incident angle, and room light. There was no significant variation in the perfusion values due to changes in camera distance between 18-27 cm in healthy skin, which agrees with a previous study in which the camera head distance was found not to affect perfusion values [7]. It should also be noted that the variation in perfusion values as a result of variations in distance, angle and room light was negligible compared to the much larger variations observed between burn wounds of different depth and healthy skin.

Previous studies support the fact that the status of the skin perfusion indicate burn wound depth. Superficial burn wounds show an initial increased perfusion which gradually declines. [8, 9]. The high perfusion in superficial burn wounds can be explained by a better preserved and more active microcirculation compared to deeper burn injuries. In superficial burns, the inflammatory reactions cause vasodilatation, increased blood flow and raise the skin metabolism. Deeper burn wounds, on the other hand, present a low initial perfusion which progressively increases. In the centre of deep burns deeper vascular structures are damaged and the circulation either restores in cases of spontaneous healing or surgery is required [8].

A strength of the protocol used in the present study is that all wounds were observed for at least 14 days before surgery and therefore, all wounds were observed long enough to be able to decide whether they were partial thickness wounds or not. This eliminates any bias related to intervention. On the other hand, this study has a number of limitations. One limitation that the LSCI technique shares with the LDI technique and other optical techniques for measurement of perfusion in burn wounds is that measurements are affected by (biological) dressing materials. In the studied patients, it was very common that pig skin was applied on parts of the wounds. We found that pig skin at least in some cases lowered the measured perfusion value substantially, probably because of its optical properties and the fact that it adds a thin layer on top of the burn wound in which 
light is partially absorbed, scattered and reflected. In the data presented in this study, we excluded all the areas of burn wounds that were covered by such dressing materials to avoid any confounding factors. It was always possible to measure without dressing material during the patients first visit to the clinic (0-24h post-burn), and therefore, a highly predictive perfusion measurement early post-burn should be aimed for. Besides dressings, the method of debridement may affect the perfusion value. Another limitation of the current study is that the results so far are based on a limited number of patients with predominantly superficial partial-thickness burns. This limits the statistical power of the study, and we could not do formal hypothesis testing at 0-24 hours post-

burn. Finally, although the results clearly suggest that LSCI can predict healing time in paediatric scald burns, the results should be considered preliminary until data from larger prospective studies based also on a larger number of deeper burns is available.

\section{Conclusion}

LSCI is a promising technique for the assessment of burn wounds as it allows for instant measurement of perfusion, it is insensitive to variations in camera distance, camera angle and room light and is easily implemented in burn care. In children with scald burns the perfusion, as measured with LSCI, in burn wounds that healed within 14 days was higher than in burn wounds that healed after 14 days or required surgery. This difference was already apparent at 0-24 hours postburn, and increased during the first week post-burn. Further prospective patient studies are required to validate how predictive the technique is for the outcome of various types of burn injuries.

Conflict of interest 
The study was supported with an equipment loan from Perimed AB. They had no involvement in the study design, in the collection, analysis and interpretation of data, in the writing of the manuscript and in the decision to submit the manuscript for publication. 
Acknowledgement

We thank Ing-Marie Jarnhed and Matilda Karlsson with the Burn Unit at Linköping University

Hospital for their help with recruitment of the patients and with the measurements.

This study was financially supported by Lions Research Fund, Linköping, Sweden.

\section{References}

[1] Devgan L, Bhat S, Aylward S, Spence RJ. Modalities for the assessment of burn wound depth. Journal of burns and wounds. 2006;5:e2.

[2] Heimbach D, Engrav L, Grube B, Marvin J. Burn depth: a review. World journal of surgery. $1992 ; 16: 10-5$.

[3] Nguyen K, Ward D, Lam L, Holland AJ. Laser Doppler Imaging prediction of burn wound outcome in children: is it possible before 48 h? Burns : journal of the International Society for Burn Injuries. 2010;36:793-8.

[4] Droog EJ, Steenbergen W, Sjoberg F. Measurement of depth of burns by laser Doppler perfusion imaging. Burns. 2001;27:561-8.

[5] Kaiser M, Yafi A, Cinat M, Choi B, Durkin AJ. Noninvasive assessment of burn wound severity using optical technology: a review of current and future modalities. Burns : journal of the International Society for Burn Injuries. 2011;37:377-86.

[6] Draijer M, Hondebrink E, van Leeuwen T, Steenbergen W. Review of laser speckle contrast techniques for visualizing tissue perfusion. Lasers in medical science. 2009;24:639-51. 
[7] Mahe G, Haj-Yassin F, Rousseau P, Humeau A, Durand S, Leftheriotis G, et al. Distance between laser head and skin does not influence skin blood flow values recorded by laser speckle imaging. Microvascular research. 2011;82:439-42.

[8] Kloppenberg FW, Beerthuizen GI, ten Duis HJ. Perfusion of burn wounds assessed by laser doppler imaging is related to burn depth and healing time. Burns : journal of the International Society for Burn Injuries. 2001;27:359-63.

[9] Cho JK, Moon DJ, Kim SG, Lee HG, Chung SP, Yoon CJ. Relationship between healing time and mean perfusion units of laser Doppler imaging (LDI) in pediatric burns. Burns : journal of the International Society for Burn Injuries. 2009;35:818-23. 
Legends for illustrations
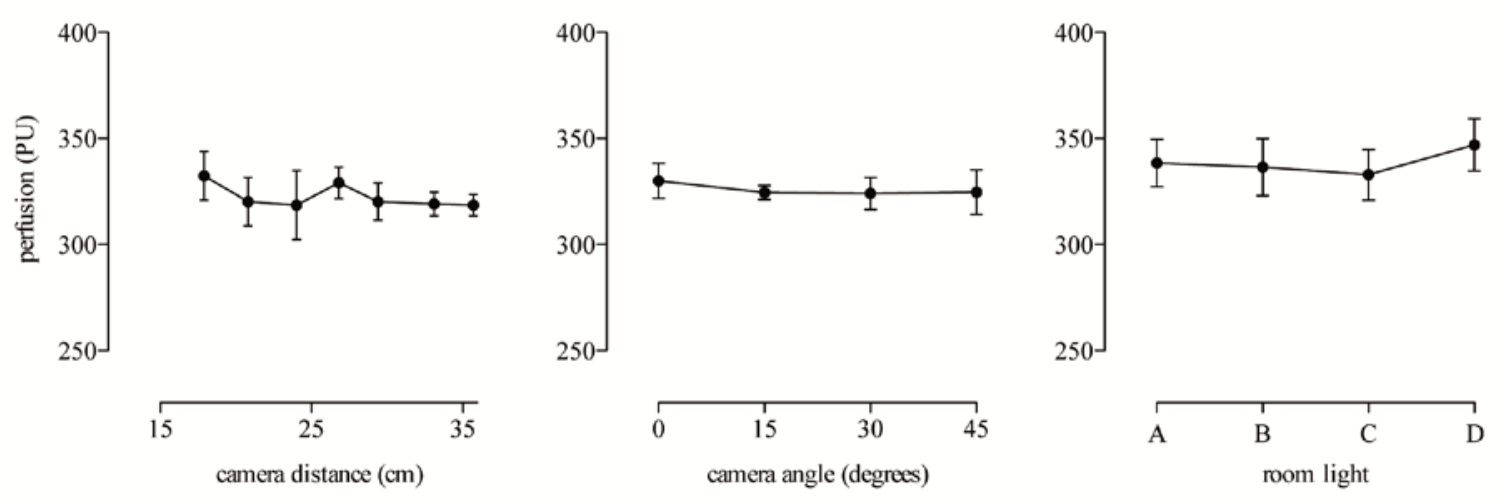

Figure 1. Effect of camera distance (left), incident angle (middle) and room light (right) on the mean $( \pm \mathrm{SD}$ ) perfusion value as measured on calibration fluid. Room light conditions were: completely dark (A), closed curtains and lights off (B), closed curtains and lights on (C) or open curtains and lights on (D).
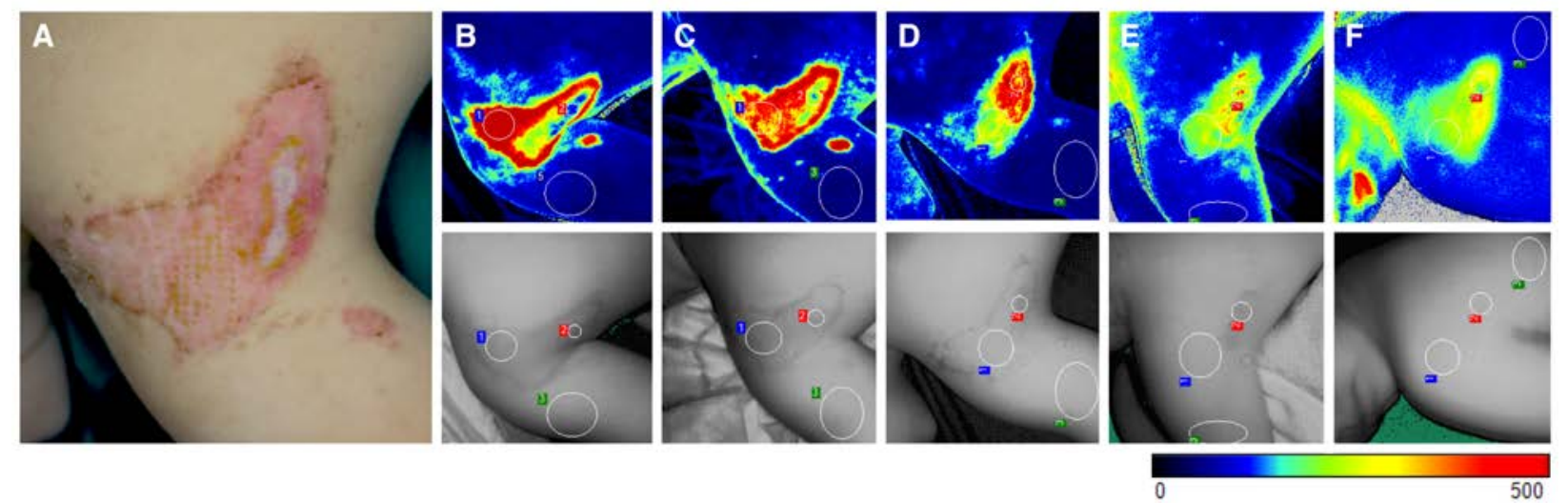

Figure 2. Photograph (A) and a series of LSCI images showing the perfusion in a partial thickness burn at (B) 3 days, (C) 6 days, (D) 9 days, (E) 15 days and (F) 21 days post-burn. A peripheral, more superficial region (ROI 1) and a central, deeper area (ROI 2) can be distinguished. ROI 3 corresponds to the perfusion in uninjured skin. 


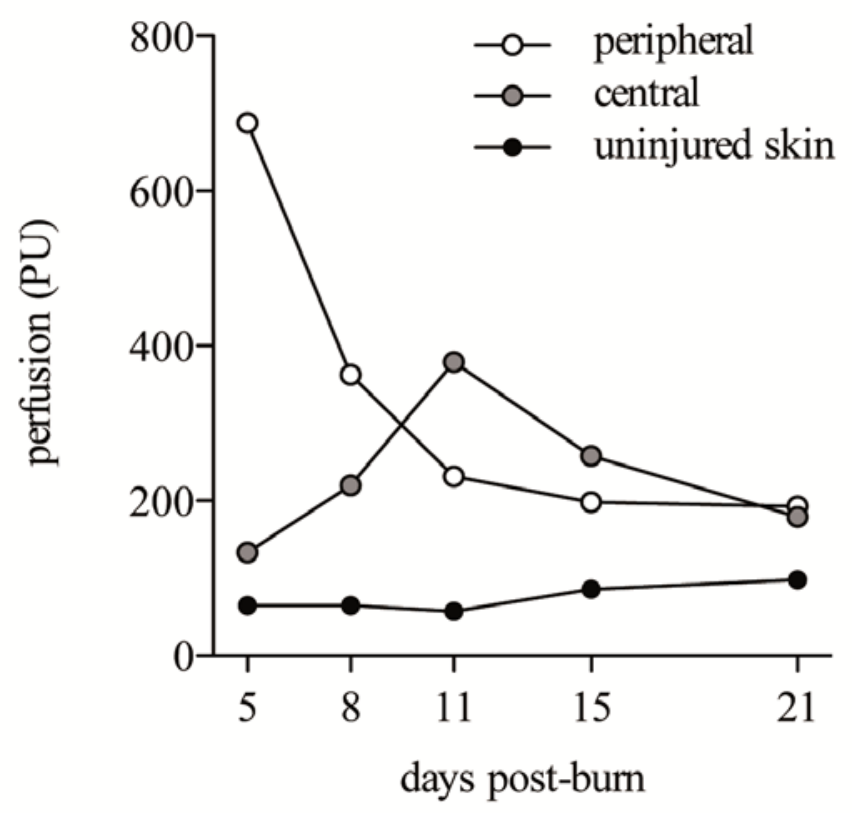

Figure 3. Change in perfusion during the first 21 days post-burn in a partial thickness scald burn of a 3-year old patient. The peripheral part of the burn was more superficial, while the central part was deeper. The perfusion in the surrounding, uninjured skin is also shown. 


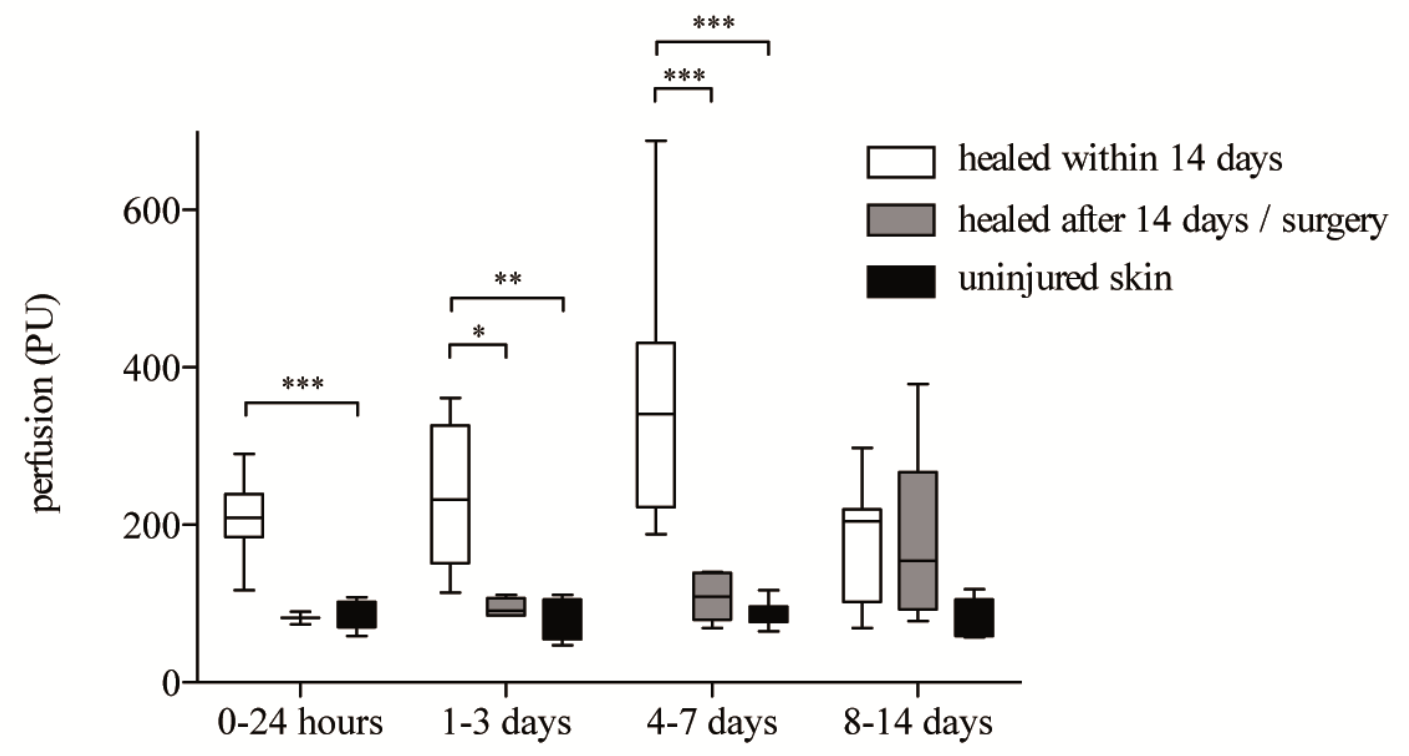

Figure 4. Mean ( \pm SD) perfusion during the first 14 days post-burn, in uninjured skin, in burns that healed within 14 days, and in burns that healed after 14 days or required surgery. Between 17 days post-burn, perfusion in burn wounds that healed within 14 days was significantly higher than in burns that healed after 14 days or required surgery and than uninjured skin. This difference disappeared during the second week post-burn. There was no significant difference between burns that healed after 14 days or required surgery and uninjured skin. * $\mathrm{p}<0.05$; ** $\mathrm{p}<0.01$ and $* * * \mathrm{p}<0.001$ 